matched second harmonic generation in urea," Phys. Status Solidi $(A)$, vol. 42, pp. K119-K 121, 1977.

[12] K. Betzler, H. Hesse, and P. Loose, "Optical second harmonic generation in organic crystals: Urea and ammonium-malate," J. Mol. Struct., vol. 47, pp. 393-396, 1978. Here, as well as in [11], all the data are reported for type II SHG. However, we think the results should refer to type $I$.

[13] C. Cassidy, J. M. Halbout, W. Donaldson, and C. L. Tang, "Nonlinear optical properties of urea," Opt. Commun., vol. 29, pp. 243-246, 1979.

[14] M. H. Hobden, "Phase-matched second harmonic generation in biaxial crystals," J. Appl. Phys., vol. 38, pp. 4365-4372, 1967.

[15] See, for example, S. K. Kurtz in Quantum Electronics, H. Rabin and C. L. Tang, Eds. New York: Academic, 1975.

[16] R. Bechmann and S. K. Kurtz in Landolt-Bornstein New Series, Gp III, vol. 2. New York: Springer-Verlag, 1969, ch. 5.

[17] This expression is given in MKS units. Note that $d$ here equals $d$ in ESU times $4 \pi / 3 \times 10^{-4}$.

[18] R. S. Adhav, Quantum Technology Ltd., private communication.

[19] G. D. Boyd and D. A. Kleinman, "Parametric interaction of focused Gaussian light beams," J. Appl. Phys., vol. 39, pp. $3597-3639,1968$.

\title{
A Theoretical and Experimental Investigation of the Modes of Optical Resonators with Phase-Conjugate Mirrors
}

\author{
JOHN AUYEUNG, STUDENT MEMBER, IEEE, D. FEKETE, DAVID M. PEPPER, STUdENT MEMBER, IEEe, AND \\ AMNON YARIV, FELLOW, IEEE
}

\begin{abstract}
We present an analysis of resonator properties for a cavity bounded by a phase conjugate mirror, which is generated by a degenerate four-wave nonlinear optical interaction. Using a ray matrix formalism to describe the conjugate mirror, resonator stability conditions are derived. Longitudinal and transverse mode characteristics are discussed. Results are compared with an experiment where laser oscillation was observed at $6943 \AA$ using carbon disulfide as the nonlinear interacting medium comprising the phase conjugate mirror.
\end{abstract}

\section{INTRODUCTION}

$\mathbf{R}$ ECENT theoretical and experimental reports deal with various aspects of optical wave phase conjugation including time-reversal in the bulk [1]-[16] and in waveguides [17]-[21], real-time holography [22] -[28], optical filtering [29], [30], amplified reflection [8]-[10], channel dispersion compensation [31], and photon echoes [32] -[35]. In this paper, we consider the case of laser oscillation in a resonator in which one of the mirrors is replaced by a phase conjugate reflector which utilizes four-wave mixing. There are several characteristics that make this resonator configuration especially attractive. The well-known "time-reversal" feature of the conjugator serves not only to compensate for various potential in-

Manuscript received March 26, 1979; revised May 21, 1979. This work was supported in part by the Army Research Office, Durham, NC and also by the Hughes Aircraft Company and Weizmann Institute.

J. AuYeung, D. Fekete, and A. Yariv are with California Institute of Technology, Pasadena, CA 91125.

D. M. Pepper was with California Institute of Technology, Pasadena, CA. He is now with Hughes Research Laboratories, Malibu, CA 90265. tracavity phase aberrating elements (e.g., gain medium phase and/or polarization distortion, poor quality optical components, etc.), but also gives rise to differences in the resonator stability criterion as compared to conventional Fabry-Perot laser resonators. Furthermore, the frequency filtering nature of the conjugator yields a type of "frequency locking" of the resonator output frequency to the frequency of the pump laser (which excited the conjugate mirror). It also renders the transverse modes present in the resonator to be degenerate in frequency, thus giving rise to a "transverse mode-locking" condition, whereby the resultant mode volume in the resonator can "fit" into an aperture (subject to the limitations imposed on the angular acceptance range of the conjugate mirror). If the effective aperture within the resonator is chosen to be the transverse gain profile, for example, then the use of a phase conjugate mirror can essentially "milk" the gain medium optimally for its stored energy. Finally, by temporally pumping the conjugate mirror, one can $Q$-switch this "phase conjugate resonator." In Section II, we present an analysis for this type of resonator which, using a ray matrix formalism and a Gaussian beam description, considers resonator stability conditions, longitudinal and transverse mode spectra, and the frequency dependence of the phase conjugate mirror. Section III deals with an experiment we performed using a ruby laser as the pump source, and carbon disulfide as the conjugator's nonlinear medium. Laser oscillation was observed in a resonator configuration which was unstable in the conventional sense. Furthermore, we observe a laser output energy that is comparable with that of a similar, conventional Fabry-Perot laser. 


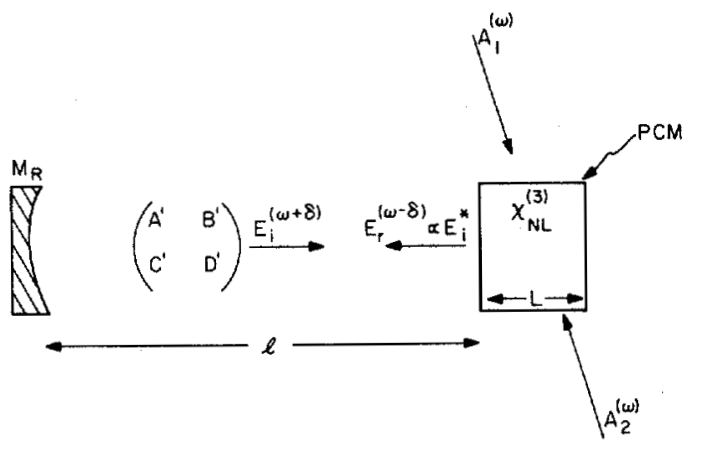

Fig. 1. The phase conjugate resonator (PCR). This general resonator is formed by surrounding some arbitrary optical components, denoted by an equivalent $A^{\prime} B^{\prime} C^{\prime} D^{\prime}$ ray matrix, by a "real" mirror (of radius $R$ ) on one end, and by a phase conjugate mirror (PCM) on the other end. For degenerate modes, $\delta=0$.

Finally, several additional observed aspects regarding the resonator will be discussed, including frequency locking of the oscillator output to the pump beams, and $Q$-switching properties due to the pulsed nature of the conjugate mirror.

\section{THEORY}

In this section, we present an analysis leading to resonator stability criteria and mode spectra for a laser resonator in which one of the mirrors is replaced by a phase conjugate mirror "PCM." The geometry of this phase conjugate resonator "PCR" is shown in Fig. 1. The PCM is assumed to be formed via a degenerate four-wave nonlinear optical interaction. The conjugate wave (of field $E_{i}$ ) $E_{r}$ is generated as a result of the simultaneous incidence of two counter-propagating plane waves $A_{1}$ and $A_{2}$ with $E_{i}$ onto a medium possessing a thirdorder nonlinear optical susceptibility, $\chi_{N L}^{(3)}$ all located within the PCM (as shown in Fig. 1). In the analysis that follows, we neglect the depletion of the pump waves $A_{1}$ and $A_{2}$ as a result of the nonlinear interaction. We also neglect diffraction losses within the PCR; and self-focusing within the PCM. We further assume that the nonlinear medium is nondispersive, and is also capable of instantaneous response.

\section{A. Matrix of the PCM (Degenerate Case)}

We first discuss a matrix formalism that describes the operation of the PCM for the case where all the interacting fields are of the same radian frequency $\omega$. The stability criterion is then derived for both one and two roundtrip self-consistent situations.

Consider a Gaussian field $E_{i}$ propagating along the $z$-axis, to be incident upon the PCM. We thus have [36]

$$
E_{i}=\mathscr{E}_{i}(\vec{r}) \exp \left[i\left(\omega t-k z-\frac{k r^{2}}{2 \rho}\right)-\frac{r^{2}}{w^{2}}\right]
$$

where $\mathscr{E}_{i}(\vec{r})$ is the complex amplitude of $E_{i}$, and $\rho$ and $w$ are the radius of curvature and the spot size of the incident field, respectively. This field can also be written as

$$
E_{i}=\varepsilon_{i}(\vec{r}) \exp \left[i\left(\omega t-k z-\frac{k r^{2}}{2 q_{i}}\right)\right] .
$$

The complex radius of curvature $q_{i}$ is defined as

$$
\frac{1}{q_{i}}=\frac{1}{\rho}-\frac{i \lambda}{\pi w^{2}} .
$$

The effect of the PCM is to "reflect" such an incident field as to yield its conjugate replica [37], leaving the wavefront and the spot size unchanged. The reflected field is

$$
E_{r} \propto \mathscr{E}_{i}^{*}(\vec{r}) \exp \left[i\left(\omega t+k z+\frac{k r^{2}}{2 \rho}\right)-\frac{r^{2}}{w^{2}}\right]
$$

which can also be expressed as

$$
E_{r} \propto \varepsilon_{i}^{*}(\vec{r}) \exp \left[i\left(\omega t+k z-\frac{k r^{2}}{2 q_{r}}\right)\right] .
$$

The reflected field complex radius of curvature subject to (3) and (4) is given by

$$
\frac{1}{q_{r}}=-\frac{1}{\rho}-\frac{i \lambda}{\pi w^{2}}=-\frac{1}{q_{i}^{*}} .
$$

An observer traveling with the reflected beam will find the spot size unchanged, but having an opposite sign for the curvature of the wavefront.

If we introduce the ray matrix formalism [36], [38], the effect of the PCM can thus be represented by the matrix

$$
M=\left(\begin{array}{cc}
A & B \\
C & D
\end{array}\right)=\left(\begin{array}{cc}
1 & 0 \\
0 & -1
\end{array}\right)
$$

with the output and input $q$-parameters related by

$$
q_{r}=\frac{A q_{i}^{*}+B}{C q_{i}^{*}+D} \text {. }
$$

Note the conjugation operation upon $q_{i}$, as opposed to the conventional formalism [36] where the input field is not conjugated. We note that this matrix also describes the reflection of rays from the conjugate mirror. That this matrix (7) and the condition given by (8) satisfy the constraint (6) can be easily verified by substitution.

It follows directly that the ordinary $A B C D$ formalism for treating the propagation of Gaussian beams through a sequence of lens-like media [38] can be applied also in the case when one of the elements is a PCM. The matrix representing the $P C M$ is given by (7). The " $q$ " parameter at any plane following the PCM is related to the input " $q$ " by

$$
q_{\text {out }}=\frac{A_{T} q_{i}^{*}+B_{T}}{C_{T} q_{i}^{*}+D_{T}}
$$

where the subscript " $T$ " implies that the matrix elements correspond to that of the resultant matrix for the given sequence of optical elements, including that of the PCM. Since all the matrices are assumed to be real, the conjugation operation imposed by (9) can be performed at any plane. We note that care must be taken when treating propagation through media described by matrices having complex elements. In this case, 
one must first evaluate the complex $q$-parameter just prior to the PCM (using the conventional formalism), apply the operations given by (7) and (8) to describe the effect of the PCM, then finally, evaluate the resultant $q$-parameter at the desired plane "after" the PCM.

\section{B. Stability Condition for One Roundtrip (Degenerate Case)}

Consider the situation sketched in Fig. 1. The resonator is bounded on one end by a mirror having a radius of curvature $R$, containing arbitrary intracavity optical components described collectively by an $A^{\prime} B^{\prime} C^{\prime} D^{\prime}$ matrix $M^{\prime}$ for optical propagation from left to right and again by an $A^{\prime \prime} B^{\prime \prime} C^{\prime \prime} D^{\prime \prime}$ matrix $\boldsymbol{M}^{\prime \prime}$ for propagation from right to left. The resonator is bounded on the other end by a PCM. In order to investigate the stability criterion for such a cavity, we apply the standard self-consistent formalism and thus demand that the complex radius of curvature of the beam reproduce itself after one roundtrip. Choosing a plane to the immediate right of the real mirror, we trace a beam that propagates to the right and get, after one roundtrip, the following matrix product:

$$
\begin{aligned}
\boldsymbol{M}_{1} & =\left(\begin{array}{ll}
A_{1} & B_{1} \\
C_{1} & D_{1}
\end{array}\right)=\left(\begin{array}{cc}
1 & 0 \\
\frac{-2}{R} & 1
\end{array}\right)\left(\begin{array}{cc}
A^{\prime \prime} & B^{\prime \prime} \\
C^{\prime \prime} & D^{\prime \prime}
\end{array}\right)\left(\begin{array}{cc}
1 & 0 \\
0 & -1
\end{array}\right)\left(\begin{array}{cc}
A^{\prime} & B^{\prime} \\
C^{\prime} & D^{\prime}
\end{array}\right) \\
& =\left(\begin{array}{ll}
1 & 0 \\
\frac{-2}{R} & 1
\end{array}\right)\left(\begin{array}{cc}
1 & 0 \\
0 & -1
\end{array}\right)
\end{aligned}
$$

where we have used the relation

$$
M^{\prime \prime} M=M\left(M^{\prime}\right)^{-1}
$$

which can be shown straightforwardly, using the reciprocity property of the group of optical elements represented by $\boldsymbol{M}^{\prime}$ (or $\boldsymbol{M}^{\prime \prime}$ ), where $\boldsymbol{M}$ is given by (7).

The above result [see (10b)] is merely a reaffirmation of the fact that an arbitrary sequence of passive and lossless optical elements followed by a PCM is equivalent to the PCM alone. This is due to the time reversal occurring at the PCM and the reciprocity of the passive components.

Now we impose self-consistency; that is, we demand that the field be reproduced at the aforementioned plane after one round trip. Using (8), this condition is

$$
\frac{1}{q}=\frac{C_{1}+D_{1} / q^{*}}{A_{1}+B_{1} / q^{*}}
$$

The result of this constraint yields the following two conditions:

$$
A_{1}+D_{1}=0
$$

and

$$
B_{1}\left[\frac{1}{\rho^{2}}+\left(\frac{\lambda}{\pi w^{2}}\right)^{2}\right]+\left(A_{1}-D_{1}\right) \frac{1}{\rho}-C_{1}=0 .
$$

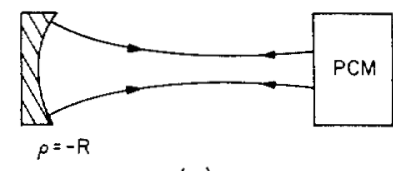

(a)

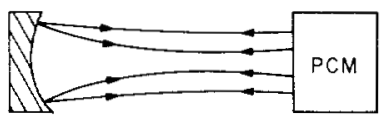

(b)

Fig. 2. Sketch of a typical allowed PCR Gaussian mode for the degenerate case and demanding self-consistent field solutions for (a) one roundtrip and (b) two roundtrips.

Upon substitution of the resultant matrix $M_{1}$ into (12), we get

$$
\rho=-R \text {. }
$$

Hence, the radius of curvature of the Gaussian beam is equal to that of the real mirror at the real mirror's plane. This conclusion is also independent of the sign of the mirror's curvature. Also, there is no dependence of the stability conditions upon the cavity length or any other optical components within the cavity. In addition, there is no constraint on the spot size (w) of the resultant mode (subject to the angular acceptance limitations of the PCM). This freedom of spot size in conjunction with the frequency degeneracy of the transverse modes at the pump frequency (to be discussed later), makes possible the notion of "spatial mode locking" of transverse modes. In Fig. 2(a), we sketch a typical Gaussian mode that is self-consistent for the one-roundtrip degenerate frequency case.

\section{Stability Condition for Two Roundtrips (Degenerate Case)}

In conventional resonators, all allowed eigenmodes are obtained by demanding a single-round trip self-consistent solution. However, for the case of a PCR, it is possible to have allowed modes which will reproduce themselves after two round trips. Furthermore, by simple ray tracing, it can be shown that, due to the time-reversing nature of the PCM, these rays will reproduce themselves after two roundtrips. Thus, for t:o roundtrips, we can use the matrix from the preceding section, forming the resultant matrix as

$$
\begin{aligned}
M_{2} & =\left(M_{1}\right)^{2} \\
& =I
\end{aligned}
$$

where $I$ is the identity matrix. We note that, since for a two roundtrip situation, we have encountered the PCM twice, the $q$-parameters have been conjugated two times and thus remain unchanged. Hence, in contrast to the single roundtrip constraints, any complex radius of curvature, (i.e., both $\rho$ and $w$ ) at the initial plane (at the real mirror) will yield a selfconsistent solution. We therefore conclude (as was the case for the one-roundtrip constraint) that the PCR is stable for any real mirror, regardless of its radius of curvature or sign, and is stable regardless of the cavity length or the intracavity optical components. 
We thus see that there exists a large range of Gaussian beam parameters that satisfy the self-consistency criterion for both the one- and two-roundtrip cases. This multitude of acceptable solutions follows from the very nature of the PCM. Since we have specified only one constraint within the resonator (in this case the radius of curvature of the real mirror), and realizing that the action of the PCM is to replicate any field incident upon its surface, there exists no unique set of $\rho$ and $w$ within the resonator. This is in contrast to conventional resonators, where, in general, the curvature is specified at two planes in space (which, therefore for stable resonators, establishes the value of the complex curvature throughout space).

We have not taken into account any additional constraints within the PCR, which can be due to several sources; one can, for example, place an aperture within the resonator. In addition, the PCM itself can possess an effective aperture due to, for example, the spatial extent of the pump beams. Another possibility can be the "effective aperture" introduced by the gain medium (i.e., the transverse gain profile). We note that in this case, the action of the PCM is to form a mode volume within the resonator that interacts with as much of the gain medium as possible, while still being above threshold. Hence, in this configuration, the PCR can be capable of most efficiently extracting the stored laser energy within a given gain medium.

In Fig. 2(b), we sketch an allowed Gaussian mode that satisfies the two-roundtrip degenerate frequency case.

\section{Ray Matrix for the PCM (Nondegenerate Case)}

We now treat the self-consistency requirement for one and two roundtrips within the PCR for the case of nondegenerate fields. Knowledge of the stability condition for nondegenerate fields is relevant to analyzing PCR "modes" that are not at the pump frequency (to be considered later). We consider the case where the pump waves exciting the PCM are both at radian frequency $\omega$, while the PCR field incident upon the PCM is at radian frequency $\omega+\delta$, where $\delta$ can be greater than or less than 0. Due to the frequency flipping nature [29] of the PCM, the conjugate field is at frequency $\omega-\delta$. The phase-matching constraint requires that each plane wave component of a given signal field incident upon the PCM to give rise to a "conjugate replica" whose $k$-vector is antiparallel to that of the input plane wave component considered. This applies to both the degenerate [8] and the nondegenerate [29] cases. Thus, the ray matrix given by (7) can also be used to describe the effect of the PCM for each plane wave component (or ray) for the nondegenerate case. However, for the nondegenerate case of an arbitrary incident wavefront, the "reflected" field no longer exactly retraces the path of the input wave, due to their difference in frequency.

This problem can be treated formally by decomposing the input field into its plane wave components, using (7) for the effect of the PCM for each of these components, changing $\omega+\delta$ to $\omega-\delta$, then finally forming the resultant superposition.

In what follows, we show that for the special case of Gaussian beams, one can form a frequency-dependent $A B C D$ "ray" matrix that properly describes the effects of the PCM. Con- sider an incident Gaussian beam of the form

$$
\begin{aligned}
E_{i}= & \&(\vec{r}) \exp \left\{i \left[\left(1+\frac{\delta}{\omega}\right) \omega t-\left(1+\frac{\delta}{\omega}\right) k z\right.\right. \\
& \left.\left.-\left(1+\frac{\delta}{\omega}\right) \frac{k r^{2}}{2 q_{i}}\right]\right\}
\end{aligned}
$$

where $k=\omega n / c$.

The "reflected" field from the PCM (located at $z=0$ ) is given by

$$
\begin{aligned}
E_{r} & \propto \mathscr{E} *(\vec{r}) \exp \left\{i \left[\left(1-\frac{\delta}{\omega}\right) \omega t+\left(1-\frac{\delta}{\omega}\right) k z\right.\right. \\
& \left.\left.-\left(1+\frac{\delta}{\omega}\right) \frac{k r^{2}}{2\left(-q_{i}^{*}\right)}\right]\right\} .
\end{aligned}
$$

This can be rewritten as

$$
\begin{aligned}
E_{r} \propto \&^{*}(\vec{r}) \exp \left\{i \left[\left(1-\frac{\delta}{\omega}\right) \omega t+\left(1-\frac{\delta}{\omega}\right) k z\right.\right. \\
\left.\left.-\left(1-\frac{\delta}{\omega}\right) \frac{k r^{2}}{2 q_{r}}\right]\right\} .
\end{aligned}
$$

This reflected field is thus recognized as being a Gaussian beam at frequency $\omega[1-(\delta / \omega)]$ and having a complex radius of curvature $q_{r}$ which can be related to $q_{i}$ by

$$
q_{r}=-q_{i}^{*} \frac{(1-\delta / \omega)}{(1+\delta / \omega)}
$$

The "ray" matrix that relates $q_{i}$ to $q_{r}$ which satisfies (15)-(18) can be represented by

$$
M=\left(\begin{array}{cc}
1-\frac{\delta}{\omega} & 0 \\
0^{*} & -\left(1+\frac{\delta}{\omega}\right)
\end{array}\right)
$$

where the operation given in (8) is to be used. We note that as $\delta \rightarrow 0,(19)$ reduces to the degenerate-case matrix given by (7). It can be shown that the effect of the PCM is to change the radius of curvature (at the output boundary of the PCM) while leaving the spot size unchanged (this follows from the frequency-flipping effect of the PCM). As discussed above, we remark that (19) applies to Gaussian beams and not to rays.

\section{E. Stability Condition for One Roundtrip (Nondegenerate Case)}

Following the discussion given for the degenerate case, we can now solve for the self-consistency constraint for the nondegenerate situation. Demanding replication after one roundtrip, we now consider an explicit resonator configuration. For the ease of calculation, we set the $\boldsymbol{M}^{\prime}$ and $\boldsymbol{M}^{\prime \prime}$ matrices of Section II-B to represent cavity spacing of length $l$. That is,

$$
M^{\prime}=M^{\prime \prime}=\left(\begin{array}{ll}
1 & l \\
0 & 1
\end{array}\right)
$$

For this choice of the cavity matrix, the total single roundtrip matrix evaluated at the real mirror plane is given by 


$$
\begin{aligned}
M_{1} & =\left(\begin{array}{cc}
1 & 0 \\
-\frac{2}{R} & 1
\end{array}\right)\left(\begin{array}{cc}
1 & l \\
0 & 1
\end{array}\right)\left(\begin{array}{cc}
1-\frac{\delta}{\omega} & 0 \\
0 & -\left(1+\frac{\delta}{\omega}\right)
\end{array}\right)\left(\begin{array}{ll}
1 & l \\
0 & 1
\end{array}\right) \\
& =\left(\begin{array}{cc}
1-\frac{\delta}{\omega} & -2 l \frac{\delta}{\omega} \\
-\frac{2}{R}\left(1-\frac{\delta}{\omega}\right) & \frac{\delta}{\omega}\left(\frac{4 l}{R}-1\right)-1
\end{array}\right)
\end{aligned}
$$

The self-consistency criterion (11) yields the same conditions [see (12a) and (12b)] as for the degenerate case. Using the resultant matrix (21) in first condition [see (12a)] gives the constraint $l=R / 2$. Substitution of this result into the second condition [see $(12 b)]$ does yield a range of $(p, w)$ pairs which satisfy the equation. However, due to the frequency-flipping nature of the PCM, any $(\rho, w)$ pair at frequency $\omega+\delta$ will not replicate itself after a second roundtrip, i.e., at frequency $\omega-\delta$. That is, there is no common $(\rho, w)$ pair that satisfies the self-consistency requirement for both $\pm \delta$ frequency components.

We thus conclude that no stable mode exists for the oneround-trip nondegenerate case.

\section{F. Stability Condition for Two Roundtrips (Nondegenerate Case)}

In considering the nondegenerate two-roundtrip stability condition, we cannot simply take the square of the singleroundtrip matrix, (21) (as was done in the degenerate frequency case). This is due to the frequency-flipping nature of the PCM. That is, upon each "reflection" off the PCM, the field changes frequency. Hence, the total two-roundtrip matrix, using (20) to describe the intracavity propagation, becomes

$$
\begin{aligned}
M_{2}= & \left(\begin{array}{ll}
A_{2} & B_{2} \\
C_{2} & D_{2}
\end{array}\right)=\left(\begin{array}{cc}
1+\frac{\delta}{\omega} & 2 l \frac{\delta}{\omega} \\
-\frac{2}{R}\left(1+\frac{\delta}{\omega}\right) & -\frac{\delta}{\omega}\left(\frac{4 l}{R}-1\right)-1
\end{array}\right) \\
& \cdot\left(\begin{array}{cc}
1-\frac{\delta}{\omega} & -2 l \frac{\delta}{\omega} \\
-\frac{2}{R}\left(1-\frac{\delta}{\omega}\right) & \frac{\delta}{\omega}\left(\frac{4 l}{R}-1\right)-1
\end{array}\right)
\end{aligned}
$$

where the second matrix has $\delta \rightarrow-\delta$, consistent with the above remark.

We note that since we have encountered the PCM twice, the conjugation operation is effectively cancelled. Thus, the applicable self-consistency criterion corresponds to that of conventional resonators [38], and is given by

$$
\frac{1}{q}=\frac{D_{2}-A_{2}}{2 B_{2}} \pm i \sqrt{-\left(\frac{D_{2}-A_{2}}{2 B_{2}}\right)^{2}-\frac{C_{2}}{B_{2}}} .
$$

Substituting the resultant matrix [see (22)] into the selfconsistency condition (23) yields $\rho=-R$; i.e., the Gaussian beam curvature at the mirror is equal that of the real mirror

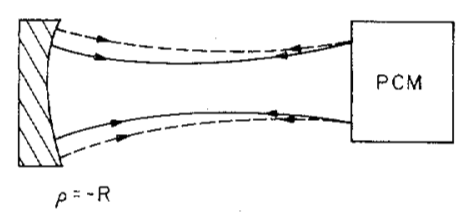

Fig. 3. Sketch of a typical allowed PCR Gaussian mode for the nondegenerate case. The solid and dashed lines correspond to a positive $(\delta>0)$ and negative $(\delta<0)$ frequency offset, respectively. Note that at the real mirror end, $\rho(\delta>0)=\rho(\delta<0)$; while at the PCM, $w(\delta>0)=w(\delta<0)$, consistent with a two-round trip self-consistent solution. Recall that for the nondegenerate case, there does not exist a solution which satisfies the single-roundtrip constraint.

radius of the curvature at each roundtrip, and is independent of the frequency offset $\delta$. However, the spot size (at the real mirror) does depend on the frequency offset and can be shown to be

$$
w=\sqrt{\frac{c}{\pi(\omega+\delta)}}\left\{\frac{1}{R}\left(\frac{1}{R}-\frac{1}{l}\right)\left[\frac{1-\frac{\delta}{\omega}\left(1-\frac{2 l}{R}\right)}{1+\frac{\delta}{\omega}\left(1-\frac{2 l}{R}\right)}\right]\right\}^{-1 / 4} .
$$

From (24) we get the following condition for stable modes to exist

$$
\begin{array}{ll}
l>R ; & \text { for } R>0 \\
\text { all } l ; & \text { for } R<0 .
\end{array}
$$

We thus conclude that for nondegenerate fields, the PCR is stable only over limited ranges of cavity length (for a given positive mirror radius). Furthermore, the radius of curvature of the mode always matches that of the real mirror; however, the spot size alternates between two values for each roundtrip [by using $\pm \delta$ in (24)] .

In Fig. 3 we sketch a typical stable Gaussian mode for the nondegenerate, two-round trip self-consistent condition.

We note that when $\delta=0,(24)$ is no longer valid since the matrix element $B_{2}=0$ [see (23)]. In this limit, the discussion presented in Section II-C applies.

\section{G. Longitudinal and Transverse PCR Mode Spectra}

In this section, we derive the PCR longitudinal and transverse mode spectra for Gaussian beams. Due to the frequencyflipping nature of the PCM, a given frequency component (e.g., at $\omega+\delta$ ) requires two roundtrips to return to its initial frequency. Alternatively, if we assume two fields to coexist (i.e., at $\omega \pm \delta$ ) we can then superpose these fields and thus require that this superposition repeat only after one roundtrip. Referring to Fig. 1, we assume that the PCR is of length and linear index, $l$ and $n_{c}$, respectively; the PCM is of length and linear index, $L$ and $n$, respectively. We represent the total field at the real mirror as

$$
E_{1}=A_{+} e^{i \theta+} \exp ^{i(\omega+\delta) t}+A_{-} e^{i \theta-} \exp ^{i(\omega-\delta) t}
$$

where $A_{ \pm}$and $\theta_{ \pm}$correspond to the magnitude (of the amplitude) and phase of the $\pm \delta$ component of the field, respectively. 
We note that $A$ and $\theta$ contain the transverse mode order $(p, q)$ of the Gaussian field [36] ; [38].

This total Gaussian field is now propagated to the right by a distance $l$ and is incident upon the PCM.

The effect of the PCM (in addition to the previously mentioned properties) is to multiply the field by a complex frequency-dependent reflectivity [29], $R_{ \pm} e^{i \phi} \pm$ given by

$$
\begin{aligned}
R_{ \pm}= & \frac{2 \pi}{n c}(\omega \mp \delta)\left|\chi_{N L}^{(3)} A_{1} A_{2} \tan (\beta L)\right| \\
& \cdot\left\{\beta^{2}+\left(\frac{n \delta}{c}\right)^{2} \tan ^{2}(\beta L)\right\}^{-1 / 2}
\end{aligned}
$$

and

$$
\phi_{ \pm}=-\frac{\pi}{2}+\eta \pm \tan ^{-1}\left[\frac{n \delta}{c \beta} \tan (\beta L)\right]
$$

where

$$
\beta=\left\{\left(\frac{2 \pi}{n c}\right)^{2}\left|\chi_{N L}^{(3)} A_{1} A_{2}\right|^{2}\left(\omega^{2}-\delta^{2}\right)+\left(\frac{n \delta}{c}\right)^{2}\right\}^{1 / 2}
$$

and $\eta$ is the phase of $\chi_{N L}^{(3)} A_{1} A_{2} \tan (\beta L)$. In (27) the ( \pm ) subscript implies that the field incident upon the PCM is of radian frequency $\omega \pm \delta$, while the "reflected" field is of radian frequency $\omega \mp \delta$, where $\omega$ is the pump frequency (of fields $A_{1,2}$ ).

The self-consistency requirement yields the following condition on the mode spectra

$$
\begin{gathered}
\tan ^{-1}\left\{\frac{2 \pi n \Delta \nu_{m}}{c \beta_{m}} \tan \left(\beta_{m} L\right)\right\}+\frac{4 \pi n_{c} l \Delta \nu_{m}}{c}+(p+q+1) \tan ^{-1} \\
\cdot\left\{\frac{-\frac{4 \pi \Delta \nu_{m}}{\omega}\left(1-\frac{2 l}{R}\right) \sqrt{1-\frac{R}{l}}}{\left[1-\left(\frac{2 \pi \Delta \nu_{m}}{\omega}\right)^{2}\left(1-\frac{2 l}{R}\right)^{2}\right]^{1 / 2}\left[2-\frac{R}{l}\right]}\right\}=m \pi
\end{gathered}
$$

where $\Delta \nu_{m}=\delta / 2 \pi$ and $(p, q)$ corresponds to the transversemode indexes of the Gaussian beam [36], [38], and $m$ is an integer.

The resultant set of PCR modes [corresponding to a mode set $(m, p, q)]$ of frequency $\omega / 2 \pi+\Delta v_{m, p, q}$ is thus given by the solutions to (28). We first see that for $m=0$ (i.e., $\Delta \nu_{m}$ $=0)$ all the transverse modes $(p, q)$ are at the same frequency (i.e., equal to the pump frequency $\omega$ ). Thus, in contrast to conventional resonators, where for a given longitudinal mode each transverse mode has a different frequency, the PCR transverse modes (for $m=0$ ) are degenerate in frequency. This gives rise to the possibility of a spatial transverse mode locking condition. If a number of transverse modes (i.e., modes with different values of $p, q$ ) exist all with $m=0$, then the resulting spatial field distribution due to the superposition of these modes will be stationary. This possibility of an oscillation field made up of a superposition of degenerate transverse modes with arbitrary waists and waist locations affords a great deal of flexibility for the mode to assume spatial

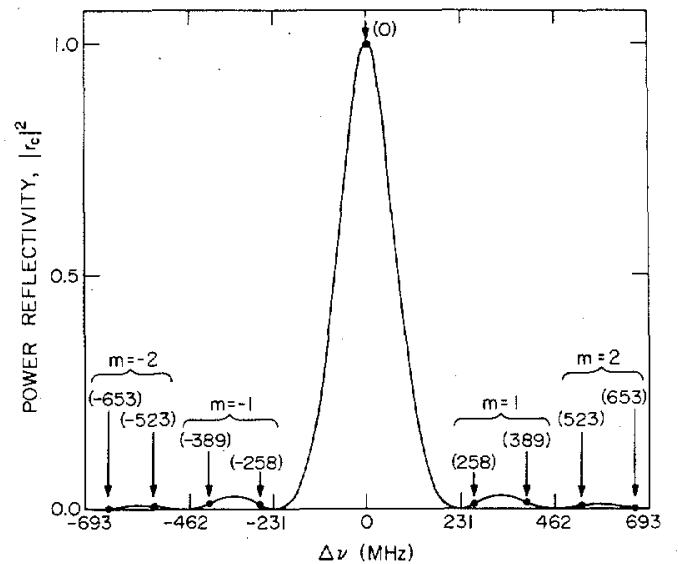

Fig. 4. PCR axial mode structure, indicated by arrows, and phaseconjugating mirrot (PCM) power reflectivity $\left|r_{c}\right|^{2}$ (after [29]) versus $\Delta \nu$, for a value of $|\kappa| L=\pi / 4 . \quad \Delta \nu=0$ corresponds to the pump frequency of the PCM. Values in parentheses indicate the frequency offset relative to the pump frequency (in $\mathrm{MHz}$ ) of several PCR modes [roots of (28)]. The transverse mode spectra (which is degenerate for $m=0$ and nondegenerate for $m \neq 0$ ) is unresolvable, and hence is not shown.

distributions which will optimally milk a given gain medium or avoid an obstruction, even under dynamic conditions.

Another property resulting from (28) is that the transverse modes ( $p, q$ pairs) belonging to higher order PCR longitudinal modes (i.e., $m \neq 0$ ) are no longer degenerate in frequency. Thus, the "mode-locking" property of the PCR holds only for $m=0$. This follows from the nonvanishing phases that are accumulated as a result of the frequency shift property of the PCM for different $p$ and $q$ (except, of course, for the "accidental degeneracy" of $p, q$ pairs where $p+q=$ constant).

We further note that due to the filter character of the PCM (i.e., the phase-matching requirement), higher order longitudinal modes will experience a smaller nonlinear reflection coefficient, thus decreasing the cavity $Q$ for these nondegenerate modes. Thus, even if $l>R$, which would allow higher order modes to exist [see (25)], the filter character will discriminate against its presence. The result is that these higher order modes $(m \neq 0)$ are less likely to oscillate. Hence, one expects essentially single-frequency operation of the PCR (i.e., $m=0$ ) given monochromatic pump waves. In Fig. 4, we plot the allowed longitudinal modes [i.e., the roots of (28)], along with the nonlinear reflection coefficient of the PCM for typical PCR conditions $\left(|\kappa| \sim \pi / 4 L, L=40 \mathrm{~cm}, l=25 \mathrm{~cm}, n_{c}=1.0\right.$, and $n=1.62$ ). These quantities, which correspond to our measured experimental parameters (to be discussed below), yield a (degenerate frequency) nonlinear reflection coefficient of $\sim 100$ percent. There exist two longitudinal modes that satisfy (28) for each nonzero value of $m$. We also note that the modes are not uniformly spaced, and that the higher order longitudinal modes have reflectivities down by almost two orders of magnitude relative to that of the $m=0$ mode. Finally, we note that the higher order transverse modes (i.e., $p, q$ pairs for $m \neq 0$ ), which are not degenerate in frequency, cannot be resolved in the figure due to their close spacing (of order $10^{-4}$ units), while the higher order transverse modes ( $p, q$ pairs) corresponding to $m=0$ are all degenerate. 


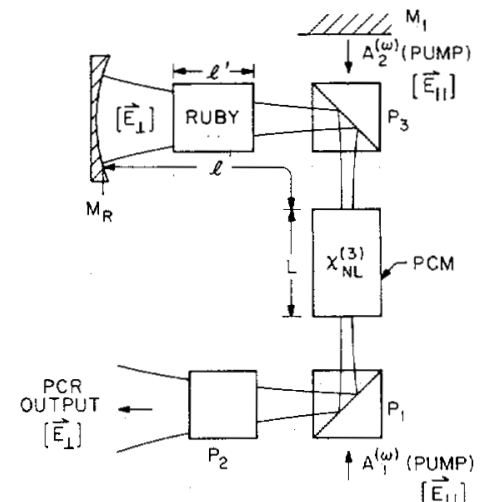

$\left[\vec{E}_{11}\right]$

Fig. 5. Schematic diagram of the experimental PCR setup. The counterpropagating pump beams $A_{1}$ and $A_{2}$ can in principle be along any arbitrary direction relative to the $\mathrm{PCR}$ optic axis; in the experiment, a collinear geometry was chosen to maximize the interaction length.

\section{EXPERIMENT}

\section{A. Description}

A sketch of the experiment designed to check some of the theoretical results is shown in Fig. 5. It consists of a "resonator" (of length $l=25 \mathrm{~cm}$ ) containing a ruby gain medium (of length $l^{\prime}=5.08 \mathrm{~cm}$ ), bounded on one end by a "real" mirror $M_{R}$ and on the other end by a "phase conjugate mirror" $\mathrm{PCM}$, (of length $L=40 \mathrm{~cm}$ ) employing $\mathrm{CS}_{2}$ as the nonlinear medium. Three different real mirror configurations were used in order to examine various aspects of the PCR, which will be discussed below. In order to obtain the largest PCM nonlinear reflection coefficient, the PCM interaction length $(L)$ was maximized using a collinear pump-signal scheme [13] with polarization discrimination used to separate the PCR fields from the pump waves. The pump waves $\left(A_{1,2}\right.$ in Fig. 5) were derived from a separate, $Q$-switched ruby laser ( $\sim 18 \mathrm{~mJ}$ in $15 \mathrm{~ns})$ operating in both a single transverse $\left(\mathrm{TEM}_{\mathrm{Do}}\right)$ and longitudinal mode. An optical delay path of $\sim 10 \mathrm{~m}$ separated the pump laser from the experimental apparatus, thus avoiding the retroreflected pump wave $\left(A_{2}\right)$ from interfering with the pump laser during the experimental measurements. The PCM was bounded by glan laser prisms to confine the PCR fields ( $s$-polarized) within the $\mathrm{CS}_{2}$, while passing the pump waves ( $\pi$-polarized). The rear glan prism $\left(P_{3}\right)$ coupled out $s$-polarized (PCR) fields which then passed through the ruby gain medium and reflected off $M_{R}$. The ruby gain medium within the PCR was aligned such that its $c$-axis was orthogonal to the s-polarized fields (thus maximizing the ruby gain coefficient for this polarization). The PCR output was monitored either from the PCM via the front glan prism $\left(P_{1}\right)$ or from a partially transmitting mirror $M_{R}$. This output field then passed through a third glan prism, which was used to eliminate any stray-reflected $\pi$-polarized fields. The total polarization rejection ratio for the PCR output calcite glan laser prisms (which were $A R$ coated and wedged), $P 1$ and $P 2$, was measured to be $\sim 10^{-12}$, thus ruling out detection of stray $\pi$-polarized fields (i.e., pump waves). When the flash-lamp pumping the gain medium within the PCR was properly synchronized with the PCM pump fields, an intense $s$-polarized pulse was detected. No output was observed when either 1) the counterpropagating pump beam, $A_{2}$ was blocked (or misaligned); or 2) the rear mirror $M_{R}$ was blocked (or misaligned). The latter test ruled out spurious effects such as self-oscillation within the PCM and regenerative amplification of depolarized or fluorescent fields; the former test ruled out ellipse rotation effects, and residual birefringence of both the optics and the $\mathrm{CS}_{2}$ cell windows.

\section{B. Resonator Stability}

In the first experiment, the real mirror $M_{R}$ (having a $2 \mathrm{~m}$ radius of curvature and being totally reflecting) was inverted, thus forming an unstable resonator in the conventional sense. In this configuration we observed oscillation of the PCR (with the PCR output coupled out from the PCM, as discussed above). The nonlinear reflection coefficient of the PCM was measured [13] to be $\sim 100$ percent for this experiment. The PCR output energy was measured to be $\sim 0.72 \mathrm{~mJ}$. This value is quite reasonable, as will be discussed below. This lends support to the theoretical result that due to the "time-reversing" nature of the PCM, the PCR should be stable regardless of the parameters of the real mirror or of the cavity length (at the degenerate frequency).

\section{PCR Energy Output}

In another experiment we compared the PCR output energy with that of an "equivalent" conventional Fabry-Perot laser. For this measurement the real mirror of the PCR was oriented to be concave with respect to the PCR fields, with the PCR output coupled out from the PCM end of the resonator. The nonlinear reflectivity of the PCM was again measured to be $\sim 100$ percent. Under these conditions, the output energy was measured to be $1.62 \mathrm{~mJ}$. This value correlates well with a measured value of $2 \mathrm{~mJ}$ obtained from the same gain medium when operated in a conventional laser resonator configuration, with the same ( $2 \mathrm{~m}$ radius) real mirror as used above, output etalon ( 60 percent reflectivity), $1.0 \mathrm{~mm}$ intracavity Mendenhall aperture, and passive $Q$-switch (cryptocyanine in methanol). The effective aperture of the PCR results from the (transverse) spatial overlap of the $\left(\mathrm{TEM}_{0_{0}}\right)$ pump beams within the PCM, which had an intensity spot size measured to be $2.2 \mathrm{~mm}$ in diameter. In fact, when the pump laser oscillated in a higher order transverse mode, the PCR output was observed to have a similar transverse character. We finally note that the greater output of the PCR using a concave, as opposed to a convex real mirror (i.e., $1.62 \mathrm{~mJ}$ versus $0.72 \mathrm{~mJ}$ ) is attributed to the fact that the former geometry sampled a greater mode volume within the ruby gain medium (as a result of the effective aperture of the PCM).

\section{PCR Frequency Spectra}

We mentioned in Section II that, for $l<R$ and $R>0$ (which corresponds to the above experimental parameters) higher order longitudinal modes do not exist. Hence, the PCR output should be frequency locked to the pump wave's frequency. (Recall that, even if $l>R$, the filter character of the PCM should also yield this frequency-locking property.) In order to verify this conjecture, the frequency spectrum of the PCR output was measured with a Fabry-Perot and compared with the pump-wave spectrum. Within the resolution of our Fabry-Perot $(\sim 150$ 


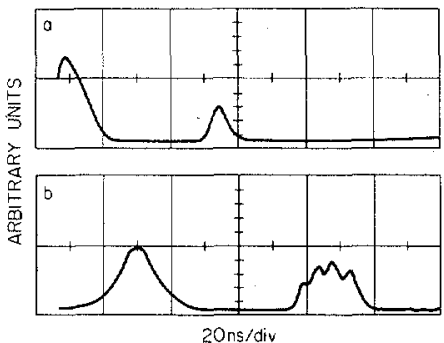

Fig. 6. Temporal evolution of the PCR output (right pulse in each trace) for a PCM power reflectivity of (a) $\sim 25$ percent and (b) $\sim 100$ percent. Note for a large reflectivity (b), the PCR output is on for a longer time interval, and also exhibits a relaxation oscillation behavior. The left pulse in each trace is that of the pump laser output.

$\mathrm{MHz}$ ), both signals were degenerate in frequency, thus experimentally confirming the above claim. The PCR Fabry-Perot spectrum also yielded no additional spectral structure. Therefore, other nonlinear processes, such as stimulated Brillouin or Raman scattering were not present.

\section{E. PCR Temporal Structure}

We now investigate the temporal output of the PCR. Since the pump laser is operated on a pulsed basis (i.e., $Q$-switched), the conjugate mirror is only "on" during the temporal overlap of the pump waves. Hence, the PCM effectively $Q$-switches the PCR. The temporal output of the PCR is given in Fig. 6(a). The left pulse is that of the pump laser, while the right pulse, delayed due to the $10 \mathrm{~m}$ delay path discussed earlier, is that of the PCR output (the amplitude of each of these pulses has been attenuated differently for visual purposes). For this measurement, the PCM reflection coefficient was measured to be $\sim 25$ percent. Increasing the PCM reflectivity to $\sim 100$ percent yields the results shown in Fig. 6(b). We note that the output pulse is on for a time longer than that corresponding to the former case. This follows from the fact that the temporal overlap of the pump beams within the PCM, which yields a time-dependent PCM reflectivity, will satisfy the PCR oscillation condition for a longer time period than that of a PCR with a smaller nonlinear gain.

We further note that the large PCM reflectivity case yields a temporal structure upon the output [see Fig. 6(b)] which is not present in the former case [see Fig. 6(a)]. We thus speculate that the temporal structure of the PCR output is attributable probably not to mode beating, but to a form of relaxation oscillation. (We note that the effect could also be due to a selffocusing phenomenon.) Recall that theory does not predict higher order modes for our experimental parameters (which occur for larger PCM reflection coefficients). Pump depletion within the PCM due to interaction with the PCR circulating intensity, in conjunction with the temporal population density variation with the PCR's ruby gain medium, apparently gives rise to such oscillations. To verify this conjecture experimentally, the temporal evolution of the pump beam $\left(A_{1}\right)$ was monitored after passage through the $\mathrm{CS}_{2}$, and was seen to also exhibit temporal fluctuations which were not present when the counterpropagating pump beam of the $\operatorname{PCM}\left(A_{2}\right)$ was misaligned, thus preventing the PCR from oscillating.

\section{CONCLUSION}

In conclusion, we have analyzed the stability criterion, longitudinal and transverse mode structure, and have demonstrated stable laser action in a novel resonator bounded by a nonlinear phase conjugating "reflective" element. The notion of frequency and spatial mode-locking of the transverse modes was discussed, which, among other features, should enable one to fully extract the stored energy within a gain medium or equivalently "fit" a mode volume through an arbitrary intracavity aperture. Several interesting properties of this device, such as the spatial, temporal, and spectral output, were shown to be consistent with that expected from such an interaction. An unstable resonator configuration in the usual sense was seen to exhibit stability and a substantial output energy. Also, timedependent saturation effects of the PCM have been observed. Finally, the PCR output energy was measured and shown to be comparable with that of a conventional resonator.

The use of a phase conjugate mirror, in addition to the abovementioned aspects, has the property of correcting for both static and dynamic phase and polarization distortions or aberrations within the resonator. Hence by coupling the PCR output from the real mirror end of the cavity, or by using a two. PCM resonator, one can thus increase the efficiency of and therefore decrease the stringent requirements for the optical components comprising the cavity.

\section{ACKNOWLEDGMENT}

D. M. Pepper and D. Fekete are thankful to the Hughes Aircraft Company and the Weizmann Institute for their support.

\section{REFERENCES}

[1] B. I. Stepanov, E. V. Ivakin, and A. S. Rubanov, "Recording twodimensional and three-dimensional dynamic holograms in transparent substances," Doklady Akademii Nauk SSSR, vol. 196, pp. 567-569, 1971; also in Sov. Phys.-Doklady, vol. 16, pp. 46-48, 1971.

[2] J. P. Woerdman, "Formation of a transient free carrier hologram in Si," Opt. Commun., vol. 2, pp. 212-214, 1970.

[3] B. Y. Zel'dovich, V. I. Popovichev, V. V. Ragul'skii, and F. S. Faisullov, "Connection between the wavefronts of the reflected and exciting light in stimulated Mandel'stam-Brillouin scattering," Pisma Zh. Eksp. Teor. Fiz., vol. 15, pp. 160-164, 1972; also in JETP Lett., vol. 15, pp. 109-113, 1972.

O. Y. Nosach, V. I. Popovichev, V. V. Ragul'skii, and F. S. Faisullov, "Cancellation of phase distortion in an amplifying medium with a 'Brillouin mirror," Pisma Zh. Eksp. Teor. Fiz., vol. 16, pp. 617-621, 1972; also in JETP Lett., vol. 16, pp. $435-439,1972$.

V. Wang and C. R. Giuliano, "Correction of phase aberration via stimulated Brillouin scattering," Opt. Lett., vol. 2, pp. 4-6, 1978.

[4] Yu. A. Anan'ev, "Possibility of dynamic correction of wave fronts," Kvant. Elektron., vol. 1, pp. 1669-1672, 1974; also in Sov. J. Quantum Electron., vol. 4, pp. 929-931, 1975.

[5] A. Yariv, "Three-dimensional pictoral transmission in optical fibers," Appl. Phys. Lett., vol. 28, pp. 88-89, 1976.

-, "Compensation of atmospheric degradation of optical beam transmission by nonlinear optical mixing," Opt. Commun., vol. 21, pp. 49-50, 1977.

P. V. Avizonis, F. A. Hopf, W. D. Bamberger, S. F. Jacobs, A Tomita, and K. H. Womack, "Optical phase conjugation in a lithium formate crystal," Appl. Phys. Lett., vol. 31, pp. 435-437, 1977.

[6] V. K. Orlov, Y. Z. Virnik, S. P. Vorotilin, V. B. Gerasinov, Y. A. Kalinin, and A. Y. Sagalovich, "Retroreflecting mirror for dynamic compensation of optical inhomogeneities," Kvant. Elektron. (Moscow), vol. 5, pp. 1389-1391, 1978; also in Sov. $J$. 
Quantum Electron., vol. 8, pp. 799-800, 1978.

H. H. Barrett and S. F. Jacobs, "Retroreflective arrays as approximate phase conjugators," Opt. Lett., vol. 4, pp. 190-192, 1979.

[7] R. W. Hellwarth, "Generation of time-reversed wavefronts by nonlinear refraction," J. Opt. Soc. Amer., vol. 67, pp. 1-3, 1977.

[8] A. Yariv and D. M. Pepper, "Amplified reflection, phaseconjugation, and oscillation in degenerate four-wave mixing," Opt. Lett., vol. 1, pp. 16-18, 1977.

[9] D. M. Bloom and G. C. Bjorklund, "Conjugate wave-front generation and image reconstruction by four-wave mixing," Appl. Phys. Lett., vol. 31, pp. 592-594, 1977.

[10] R. L. Abrams and R. C. Lind, "Degenerate four-wave mixing in absorbing media," Opt. Lett., vol. 2, pp. 94-96, 1978 and vol. 3, p. $205,1978$.

A. Elci and D. Rogovin, "Phase conjugation in nonlinear molecular gases," Chem. Phys. Lett., vol. 61, pp. 407-409, 1979.

[11] P. F. Liao and D. M. Bloom, "Continuous-wave backward-wave generation by degenerate four-wave mixing in ruby," Opt. Lett., vol. 3, pp. 4-6, 1978 and references therein.

[12] S. M. Jensen and R. W. Hellwarth, "Observation of the timereversed replica of a monochromatic optical wave," Appl. Phys. Lett., vol. 32, pp. 166-168, 1978.

[13] D. M. Pepper, D. Fekete, and A. Yariv, "Observation of amplified phase-conjugate reflection and optical parametric oscillation by degenerate four-wave mixing in a transparent medium," Appl. Phys. Lett., vol. 33, pp. 41-44, 1978.

[14] E. E. Bergmann, I. J. Bigio, B. J. Feldman, and R. A. Fisher, "High-efficiency pulsed $10.6 \mu \mathrm{m}$ phase-conjugate reflection via degenerate four-wave mixing," Opt. Lett., vol. 3, pp. 82-84, 1978.

R. C. Lind, D. G. Steel, M. B. Klein, R. C. Abrams, C. R. Giuliano, and R. K. Jain, "Phase conjugation at $10.6 \mu \mathrm{m}$ by resonantly enhanced degenerate four-wave mixing," Appl. Phys. Lett., vol. 34, pp. 457-459, 1979.

A. Tomita, "Phase conjugation using gain saturation of a Nd: YAG laser," Appl. Phys. Lett., vol. 34, pp. 463-464, 1979.

[15] D. Grischkowsky, N. S. Shiren, and R. J. Bennett, "Generation of time-reversed wave fronts using a resonantly enhanced electronic nonlinearity," Appl. Phys. Lett., vol. 33, pp. 805-807, 1978.

[16] J. P. Huignard, J. P. Herriau, P. Aubourg, and E. Spitz, "Phaseconjugate wavefront generation via real-time holography in $\mathrm{Bi}_{12} \mathrm{SiO}_{20}$ crystals," Opt. Lett., vol. 4, pp. 21-23, 1979.

[17] A. Yariv, J. AuYeung, D. Fekete, and D. M. Pepper, "Image phase compensation and real-time holography by four-wave mixing in optical fibers," Appl. Phys. Lett., vol. 32, pp. 635-637, 1978.

[18] S. M. Jensen and R. W. Hellwarth, "Generation of time-reversed waves by nonlinear refraction in a waveguide," Appl. Phys. Lett., vol. 33, pp. 404-405, 1978.

[19] J. AuYeung, D. Fekete, D. M. Pepper, A. Yariv, and R. K. Jain, "Continuous backward-wave generation by degenerate four-wave mixing in optical fibers," Opt. Lett., vol. 4, pp. 42-44, 1979.

[20] R. W. Hellwarth, "Theory of phase conjugation by stimulated scattering in a waveguide,"J. Opt. Soc. Amer., vol. 68, pp. 1050$1056,1978$.

[21] R. W. Hellwarth, "Theory of phase-conjugation by four-wave mixing in a waveguide," IEEE J. Quantum Electron., vol. QE-15, pp. 101-109, Feb. 1979.

[22] H. J. Gerritsen, "Nonlinear effects in image formation," Appl. Phys. Lett., vol. 10, pp. 239-241, 1967.

[23] D. L. Staebler and J. J. Amodei, "Coupled-wave analysis of holographic storage in $\mathrm{LiNbO}_{3}$, , J. Appl. Phys., vol. 43, pp. 1042$1049,1972$.

[24] Y. I. Ostrovskii, V. G. Sidorovich, D. I. Stasel'ko, and L. V. Tanin, "Dynamic holograms in sodium vapor," Pisma $2 h$. Tekh. Fiz., vol. 1, pp. 1030-1033, 1975; also in Sov. Tech. Phys. Lett., vol. 1 , pp. 442-443, 1975.

[25] J. P. Huignard and F. Micheron, "High-sensitivity read-write volume holographic storage in $\mathrm{Bi}_{12} \mathrm{SiO}_{20}$ and $\mathrm{Bi}_{12} \mathrm{GeO}_{20}$ crystals," Appl. Phys. Lett., vol. 29, pp. 591-593, 1976.

[26] A. Yariv, "Four-wave nonlinear optical mixing as real time hologtaphy," Opt. Commun., vol. 25, pp. 23-25, 1978.

[27] E. V. Ivakin, I. P. Petrovich, and A. S. Rubanov, "Self-diffraction of radiation by light-induced phase gratings," Kvant. Elektron., vol. 1, pp. 96-102, 1973; also in Sov. J. Quantum Electron., vol. 3 , pp. 52-55, 1973.

E. V. Ivakin, I. P. Petrovich, A. S. Rubanov, and B. I. Stepanov, "Dynamic holograms in amplifying medium," Kvant. Elektron. (Moscow), vol. 2, pp. 1556-1558, 1975; also in Sov. J. Quantum Electron., vol. 5, pp. 840-841, 1975.

[28] D. M. Pepper, J. AuYeung, D. Fekete, and A. Yariv, "Spatial convolution and correlation of optical fields via degenerate fourwave mixing," Opt. Lett., vol. 3, pp. 7-9, 1978.

[29] D. M. Pepper and R. L. Abrams, "Narrow optical bandpass filter via nearly degenerate four-wave mixing," Opt. Lett., vol. 3, pp. 212-214, 1978.

[30] J. Nilsen and A. Yariv, "Nearly degenerate four-wave mixing applied to optical filters," Appl. Opt., vol. 18, pp. 143-145, 1979.

[31] A. Yariv, D. Fekete, and D. M. Pepper, "Compensation for channel dispersion by nonlinear optical phase conjugation," Opt. Lett., vol. 4, pp. 52-54, 1979.

[32] C. V. Heer and P. F. McManamon, "Wavefront correction with photon echoes," Opt. Commun., vol. 23, pp. 49-50, 1977.

[33] N. S. Shiren, "Generation of time-reversed optical wave fronts by backward-wave photon echoes," Appl. Phys. Lett., vol. 33, pp. 299-300, 1978.

[34] N. C. Griffen and C. V. Heer, "Focusing and phase conjugation of photon echoes in Na vapor," Appl. Phys. Lett., vol. 33, pp. 865-866, 1978.

M. Fujita, H. Nakatsuka, H. Nakanishi, and M. Matsuoka, "Backward echo in two-level systems," Phys. Rev. Lett., vol. 42, pp. 974-977, 1979.

[35] A. Yariv and J. AuYeung, "Transient four-wave mixing and realtime holography in atomic systems," IEEE J. Quantum Electron., vol. QE-15, pp. 224-228, Apr. 1979.

[36] See, for example, A. Yariv, Quantum Electronics, 2nd ed. New York: Wiley, 1975, chs. 6 and 7.

[37] A. Yariv, "Phase conjugate optics and real-time holography," IEEE J. Quantum Electron., vol. QE-14, pp. 650-660, Oct. 1978. B. Y. Zel'dovich, N. F. Pilipelskii, V. V. Ragul'skii, and V. V. Shkunov, "Wavefront reversal by nonlinear optics methods," Kvant. Elektron. (Moscow), vol. 5, pp. 1800-1803, 1978; also in Sov. J. Quantum Electron., vol. 8, pp. 1021-1022, 1978.

[38] See, for example, A. Yariv, Introduction to Optical Electronics, 2nd ed. New York: Holt, Rinehart, and Winston, 1976. 